\title{
28 Research Square \\ Prognostic significance of NDRG2 combined with EGFR patients with lung adenocarcinoma
}

\section{Bo Yang ( $\square$ yangbochest@126.com )}

Tianjin First Central Hospital https://orcid.org/0000-0003-0052-048X

Xiao-Ping Li

Tianjin First Central Hospital

\section{Hong-Gang Zhou}

Nankai University College of Pharmacy

\section{Tao Jiang}

Fourth Military Medical University

\section{Ting Xiao}

Nankai University College of Pharmacy

\section{Xiao-He Li}

Nankai University College of Pharmacy

\section{Liang Zhang}

Tianjin First Central Hospital

\section{Lei Li}

Tianjin First Central Hospital

Wen-Chen Wang

PLA Army General Hospital

\section{Wei-Dong Zhang}

Tianjin First Central Hospital

\section{Research article}

Keywords: N-MYC downstream-regulated gene 2, Epidermal growth factor receptor, Lung adenocarcinoma, Prognosis

Posted Date: March 4th, 2020

DOI: https://doi.org/10.21203/rs.2.14181/v3

License: (9) (1) This work is licensed under a Creative Commons Attribution 4.0 International License. Read Full License 


\section{Abstract}

Background: N-myc downstream-regulated gene 2 (NDRG2) plays a substantial role in lung adenocarcinoma (LUAD). Epidermal growth factor receptor (EGFR) mutation could significantly improve prognosis in patients with LUAD. In this study, we aimed to elucidate the prognostic value of NDRG2/EGFR in patients with LUAD.

Methods: Immunohistochemistry, western blotting, and real-time polymerase chain reaction (RT-PCR) were conducted to detect the expression levels of NDRG2 protein. Associations between NDRG2/EGFR expression and clinicopathological characteristics of patients with LUAD were examined as well. Serum levels of carcinoembryonic antigen (CEA) were tested prior to treatments. Patients' overall survival (OS) was assessed by the Kaplan-Meier method. Multivariate Cox regression analysis was carried out to investigate the effects of patients' demographic characteristics on overall survival .

Results: The expression of NDRG2 was significantly decreased in patients with LUAD. The expression of NDRG2 was positively correlated with the levels of CEA and EGFR. Advanced stages were significantly associated with low expression of NDRG2. We found that the patients in the NDRG2-high/EGFR(+) group had the best outcomes, while the patients in the NDRG2-low/EGFR(-) group had the worst outcomes. Cox regression analysis showed that NDRG2-low/EGFR(+), NDRG2-high/EGFR(+), and vascular invasion were independent prognostic factors of LUAD.

Conclusion: NDRG2 and EGFR should be considered in patients with LUAD.

\section{Background}

Lung cancer remains the leading cause of cancer-related death worldwide, accounting for $19.4 \%$ of cancer mortality among adults [1]. Among lung cancers, small-cell lung cancers represent approximately $15 \%$ of the cases and non-small cell lung cancers (NSCLCs) approximately 85\%. NSCLC includes lung adenocarcinoma (LUAD), which is the most prevalent subtype of lung cancer [2]. Only approximately $20 \%$ of patients with NSCLC can be potentially treated by resection, and the remaining are diagnosed in advanced stages [3]. Despite advances accomplished in terms of early detection and standard treatments such as surgery, chemotherapy, radiotherapy, and iodine-125 (125I) brachytherapy, the overall survival (OS) of NSCLC still remains poor [3, 4].

Targeted therapy, such as tyrosine kinase inhibitors (TKIs), has recently emerged as a new therapeutic approach for patients with advanced NSCLC, especially for LUAD harboring EGFR mutations, showing better progression-free survival (PFS) compared with wild type tumors $[5,6]$. Nevertheless, after surgical resection, several patients eventually relapse due to inevitable drug resistance, and patients lacking driver oncogene aberrations are still treated with traditional regimens, with poor outcomes [7]. Hidayat et al. found that FBXW7 expression in CD133positive cells was increased, and c-MYC expression was decreased in gefitinib-resistant tumors from PC9 cells in mice and in nine out of 14 tumor specimens from patients with EGFR-mutant NSCLC and acquired resistance to gefitinib [8]. Another study reported that hyperprogressive disease (HPD) promptly leads to death in patients harboring EGFR exon 20 insertion mutation and MYC amplification [9]. Consequently, the interaction between MYC and EGFR may play a significant role in malignant tumor cells, involving cell proliferation, invasion, and metastasis.

The N-myc downstream-regulated gene (NDRG) family consists of four members: NDRG1, NDRG2, NDRG3, and NDRG4 [10]. A previous study showed that NDRG2 exerts important functions in cell differentiation and tumor suppression. Researches revealed that DNA damage, hypoxia, and glucocorticoids promoted NDRG2 expression, 
and NDRG2 can be transcriptionally activated by p53 and HIF1-a [11]. Decreased expression of NDRG2 has been found in several types of human cancers, such as lung cancer, bladder cancer, colon cancer, pancreatic cancer, thyroid cancer, glioblastoma, melanoma, and meningioma; besides, NDRG2 was found as a candidate tumor suppressor gene [12]. Although a previous analysis revealed that NDRG2 might serve as a novel prognostic marker in human lung cancer, its prognostic value in LUAD needs to be further elucidated. In addition, the association between NDRG2 expression and EGFR mutation still remains elusive.

Accordingly, the identification of novel prognostic markers for LUAD is urgently required. The present study examined the prognostic value of the combined detection of NDRG2/EGFR in patients with LUAD.

\section{Methods}

\section{Patients}

A total of 89 LUAD patients were prospectively enrolled in this cohort study at the Tianjin First Central Hospital of Nankai University between June 2013 and June 2014 (52 men and 37 women; mean age, 65.6 years old; range of age, 38-86 years old). None of the patients had received chemotherapy, radiotherapy, and/or immunotherapy before sampling. The clinical and pathologic characteristics of the patients with LUAD are listed in Table 1. Those individuals who smoked at least 1 cigarette per day for over 1 year were defined as smokers. Tumor staging and grading were according to the 8th edition of the Union for International Cancer Control (UICC) TNM classification of malignant tumors. Hematoxylin and eosin (H\&E) staining of tissue slides was performed and verified by two boardcertified pathologists. Among the patients, 34 cases underwent curative-intent surgery; in addition, 55 patients with advanced LUAD were treated with iodine-125 ( $\left.{ }^{125} \mathrm{l}\right)$ brachytherapy. Patients' preoperative workup (e.g., positron emission tomography (PET)/computed tomography (CT), cardiac ultrasound, and lung function) was examined to exclude those cases with secondary lung cancer and those with systemic disease (Figure 1). Normal tissues were removed from at least $5 \mathrm{~cm}$ away from the edge of the tumors.

Immunohistochemistry

In the present study, formalin-fixed paraffin-embedded tissue sections (thickness, 4- $\mu \mathrm{m}$ ) were used for detecting the expression of NDRG2. Tissue sections were dewaxed, rehydrated, antigen-retrieved, and cooled to room temperature. The sections were incubated with mouse monoclonal anti-NDRG2 antibody (Abcam, Cambridge, UK) at $4^{\circ} \mathrm{C}$ overnight, rinsed with phosphate-buffered saline (PBS), and incubated with horseradish peroxidase (HRP)labeled goat anti-mouse secondary antibody for $60 \mathrm{~min}$. NDRG2 expression was revealed using 3,3'diaminobenzidine (DAB) as the chromogen. Negative control was performed by replacing the primary antibody with normal mouse serum. The brown or yellow staining was identified as a positive expression. The total staining score of 0-12 was considered in a semi-quantitative manner and stratified as follows: negative (-, range of score: 01 ), weak (+, range of score: $2-4)$, moderate (++, range of score: 5-8), or strong (+++, range of score: $9-12)$. The tumor specimens were divided into the low-expression group (range of score: 0-4) and the high-expression group (range of score: 5-12) [12].

Western blot analysis

Total protein concentration was measured by the bicinchoninic acid (BCA) assay kit. The proteins were separated by $10 \%$ sodium dodecyl sulfate-polyacrylamide gel electrophoresis (SDS-PAGE) and transferred onto polyvinylidene fluoride (PVDF) membranes. The membranes were incubated with mouse anti-human NDRG2 
antibody (1:500, Abcam, Cambridge, MA, USA), CyclinD3 (1:1000, Cell Signaling Technology, Inc., Danvers, MA, USA), SOCS1 (1:500, Santa Cruz Biotechnology, Santa Cruz, CA, USA), and GAPDH (1:1000, Beyotime Institute of Biotechnology, Haimen, China) at $4^{\circ} \mathrm{C}$ overnight after being blocked with $5 \%$ non-fat milk for $1 \mathrm{~h}$. $\beta$-actin was used as internal control. The membranes were washed and incubated with HRP-conjugated secondary antibody (Santa Cruz Biotechnology Inc., Dallas, TX, USA). The blots were visualized using an enhanced chemiluminescence kit (Amersham Pharmacia Biotech, Arlington Heights, IL, USA) according to the manufacturer's instructions. Each experiment was performed in triplicate.

Real-time polymerase chain reaction (RT-PCR)

The total RNA was extracted from the fresh tissues using TRIzol reagent (Invitrogen, Carlsbad, CA, USA). The sequences of the primers were: NDRG2, forward-5'-ATG GCG GAG CTG CAG GAG GTC-3', and reverse-5'-AAC AAG GGC CAT TCA ACA GGA GAC-3'; GAPDH, forward-5'-GCC TCA AGA TCA GCA AT-3' and reverse-5'-AGG TCC ACC ACT GAC ACG TT-3'. The RT-PCR conditions were: denaturation at $94^{\circ} \mathrm{C}$ for $30 \mathrm{~s}$, annealing at $58^{\circ} \mathrm{C}$ for $30 \mathrm{~s}$, and extension at $72^{\circ} \mathrm{C}$ for $40 \mathrm{~s}$. The relative mRNA expression of NDRG2 was calculated by the $-2^{\Delta \Delta \mathrm{Ct}}$ method. Each experiment was undertaken in triplicate.

\section{Measurement of parameters}

Blood samples were collected before initiation of treatment. Patients' blood type was examined by measuring the serum concentrations. CEA was measured routinely at the hospital's central laboratory with human carcinoembryonic antigen ELISA kit (Abcam) .

Statistical analysis

Categorical data were expressed as frequencies or percentages. Continuous data were presented as means \pm standard deviations (SD). Differences between categorical groups were investigated by the chi-square test or Fisher's exact test. The differences in means between groups were analyzed using the Mann-Whitney U-test or Student's t-test. Associations between two variables were quantified using Spearman's rank correlation coefficient. The OS rate was evaluated using the Kaplan-Meier method, and differences between the groups were assessed using the log-rank test. Multivariable analysis was conducted using Cox's proportional hazards regression model to investigate the effects of patients' demographic characteristics on OS. All statistical tests were two-sided, and P < 0.05 was considered statistically significant.

\section{Results}

NDRG2 was downregulated in LUAD, SOCS1, and CyclinD3 compared with normal tissues

NDRG2 protein was mainly found in the cytoplasm, and a weak expression could be found in a limited number of cell nuclei (Figure $2 \mathrm{~A}-\mathrm{C}$ ). The expression of NDRG2 at the protein (Figure $2 \mathrm{D}$ ) and mRNA (Figure 2 E) levels in LUAD was significantly lower compared with normal tissues.

To evaluate the expression of NDRG2, SOCS1, and CyclinD3 in different stages of LUAD, we divided 89 pairs of normal and LUAD samples into four groups according to the disease stage. There were $24,15,10$, and 40 samples in stages I, II, III, and IV, respectively. The expression of NDRG2 and SOCS1 gradually decreased as the 
LUAD stage increases (Figure 3 B-C), while the expression of CyclinD3 gradually increased as the LUAD stage increases (Figure $3 \mathrm{D}$ ).

Relationship between the expression of NDRG2 and clinicopathological features of patients with LUAD

As shown in Figure 4, the expression level of NDRG2 was associated with CEA $(P<0.001)$.

As shown in Table 2, the expression level of NDRG2 was notably higher in LUAD tissues in stages I-II than that in stage III-IV $(\mathrm{P}<0.001)$. In addition, the frequencies of no vascular invasion and EGFR positivity $(+)$ were significantly higher in patients with high expression of NDRG2 than that in patients with low expression of NDRG2 ( $<<0.001$ and 0.001 , respectively). There were no associations between expression levels of NDRG2 and other clinicopathological features, including age, sex, smoking history, and blood type $(P>0.05)$.

Regarding the 34 patients who underwent surgery, the expression level of NDRG2 was significantly higher in stage I-II than that in stage III-IV $(P=0.028)$. The frequencies of no vascular invasion and EGFR positivity $(+)$ were higher in patients with high expression of NDRG2 than that in the patients with low expression of NDRG2 (0.008 and 0.030 , respectively).

Prognostic implications of NDRG2 and EGFR expression

Based on the clinicopathological features of the patients with LUAD, as well as the expression levels of NDRG2, EGFR, and CEA, the survival was analyzed by the Kaplan-Meier method (Figure 5). The results showed that iodine125 radioactive seeds brachytherapy for advanced LUAD with high expression level of NDRG2 led to significantly higher OS than in LUAD with low expression level $(P=0.0261$, Figure $5 \mathrm{~A})$. patients with LUAD, EGFR $(+)$, and CEA < $2.0 \mathrm{ng} / \mathrm{ml}$ had higher OS $(\mathrm{P}<0.0001,0.0314$, Figure $5 \mathrm{~B}-\mathrm{C})$. In addition, in operated patients with high expression of NDRG2 (Figure $5 \mathrm{E}$ ), EGFR(+) (Figure $5 \mathrm{~F}$ ), and CEA $<2.0 \mathrm{ng} / \mathrm{ml}$ (Figure $5 \mathrm{G}$ ), higher OS was noted $(\mathrm{P}=0.0022,<$ 0.0001 and 0.013 , respectively).

According to the conjoined expressions of NDRG2/EGFR, the subjects were categorized into four groups: NDRG2low/EGFR-negative(-), NDRG2-low/EGFR-positive(+), NDRG2-high/EGFR-negative(-), and NDRG2-high/EGFRpositive(+). The association between the co-expression of NDRG2/EGFR and OS was tested by the Kaplan-Meier method. In these four groups, iodine-125 radioactive seeds brachytherapy for advanced LUAD patients in the NDRG2-high/EGFR(+) group was accompanied by the best prognosis during the 5-year follow-up $(P<0.0001$, Figure $5 \mathrm{D})$, and the same results were observed in operated patients $(P=0.0002$, Figure $5 \mathrm{H})$.

Cox regression analysis

As shown in Table 3, NDRG2-low/EGFR(+) (hazard ratio (HR)=6.508; 95\% confidence interval (Cl), 2.619-16.174; $\mathrm{P}<0.001)$, NDRG2-high/EGFR(+) (HR=3.519; 95\% Cl, 1.384-8.949; $\mathrm{P}=0.008)$, and vascular invasion $(\mathrm{HR}=4.480$; $95 \% \mathrm{Cl}, 2.291-8.760 ; \mathrm{P}<0.001)$ were independent prognostic factors of OS.

\section{Discussion}

To improve the prediction of lung cancer survival, several tumor markers (e.g., CEA) have been assessed and extensively used $[13,14]$, but each marker has its own specificity and sensitivity, which might lead to limitations in prognostic ability. The combined detection of tumor markers may be of great importance for improving the prediction of lung cancer survival. 
MYC influences growth, proliferation, differentiation, and apoptosis of cancer cells through regulating the expression of numerous genes, including SOCS1 and CyclinD3 [15]. In addition, MYC governs events associated with tumor progression, including genetic stability, migration, and angiogenesis [16]. Two human cDNAs, encoding NDRG3 and NDRG4, are homologous to NDRG1. These two genes, together with NDRG1 and a previously deposited cDNA (designated NDRG2), constitute the NDRG gene family [17]. Previous studies reported that NDRG2 was associated with human lung cancer, and the decreased expression of NDRG2 was correlated with a worse outcome of lung cancer patients $[12,18]$. Similarly, the results of the present study showed that the high expression level of NDRG2 in LUAD patients was significantly associated with the early TNM stage and negative vascular invasion. The low expression level of NDRG2 showed a lower OS than the high expression level of NDRG2. The above-mentioned findings indicate that NDRG2 may play a pivotal role in the development of LUAD. Accordingly, the expression of SOCS1, a negative regulator of cytokine signaling, was decreased along with increasing tumor stage, while the expression of CyclinD3, which is involved in cell cycle progression, was increased with increasing tumor stage. Those results are supported by previous studies in lung cancer $[19,20]$.

CEA was first described in 1965 by Gold and Freedman as an antigen present in gastrointestinal carcinoma cells $[14,21]$. A number of researches demonstrated the prognostic value of preoperative CEA levels as a classical marker for LUAD $[22,23]$. In the present study, we, for the first time, showed that CEA levels $>2.0 \mathrm{ng} / \mathrm{ml}$ were associated with the low expression level of NDRG2, and a lower OS was found compared with CEA levels $<2.0$ $\mathrm{ng} / \mathrm{ml}$. The study indicates that CEA levels $<2.0 \mathrm{ng} / \mathrm{ml}$ might indicate a good prognosis for LUAD.

Research revealed that EGFR mutations in circulating tumor DNA (ctDNA) predicted a better PFS, in particular in advanced patients with NSCLC treated by EGFR-TKIs. KRAS mutations in ctDNA indicated a worse PFS and OS in patients treated by chemotherapy [24]. Another study demonstrated that blood, in particular serum, is an appropriate substitute when tumor tissue is absent or insufficient for testing EGFR mutations to guide EGFR-TKIs treatment in patients with NSCLC [25]. In the present research, we reported the EGFR mutation status in patients with adenocarcinoma and its correlation with the expression level of NDRG2. Mutant EGFR expression was positively correlated with higher OS in the presence of a high expression level of NDRG2.

MYC and EGFR have been identified as potential biomarkers that can predict the efficacy of targeted therapy [26, 27]. We, herein, analyzed the prognostic value of the combined detection of NDRG2 and EGFR for LUAD. We found that the patients in the NDRG2-high/EGFR(+) group had the best outcome, while the patients in the NDRG2low/EGFR(-) group had the worst. Cox regression analysis revealed that NDRG2-low/EGFR(+), NDRG2high/EGFR(+), and vascular invasion were independent prognostic factors of OS (Table 3 ).

This study has several limitations. First, it was a retrospective study performed at a single center. Second, the sample size was very limited. The present study, in itself, cannot be used to change clinical practice and to propose the use of a new test. Those results have to be validated using a large sample size. Hence, further study is required to explore the putative association between NDRG2/EGFR level and OS in patients with NSCLS.

\section{Conclusions}

In summary, this study reported the different expression levels of NDRG2 in patients with LUAD. In addition, for the first time, the relationship between NDRG2/EGFR expression and clinicopathological characteristics of patients with LUAD, especially prognosis status, was investigated. NDRG2/EGFR can be used as a novel prognostic biomarker for patients with LUAD. 


\section{Abbreviations}

NDRG2: N-Myc downstream-regulated gene2

LUAD: Lung adenocarcinoma

EGFR: Epidermal growth factor receptor

CEA: Carcinoembryonic antigen

NSCLC: Non-small cell lung cancer

TKIs: Tyrosine kinase inhibitors

HPD: Hyperprogressive disease

HIF1-a: Hypoxia-inducible factor (HIF)1-a

PBS: Phosphate-buffered saline

DAB: 3,3'-Diaminobenzidine

SDS-PAGE: Sodium dodecyl sulfate-polyacrylamide gel electrophoresis

PVDF: Polyvinylidene fluoride

RT-PCR: Reverse transcription-polymerase chain reaction

HRP: Horseradish peroxidase

SOCS1: Suppressor of cytokine signaling 1

GAPDH: Glyceraldehyde-3-phosphate dehydrogenase

SD: Standard deviation

ctDNA: circulating tumor DNA

TNM: Tumor lymph node metastasis

OS: Overall survival

PFS: Progression-free survival

SE: Standard error

HR: Hazard ratio

Cl: Confidence intervals

\section{Declarations}


Acknowledgment

We thank Prof. Lei Liu, Lan Wu, Wei Gao, and Wen Hou from the Department of Science and Education of Tianjin First Central Hospital of Nankai University for their kind technical assistance.

\section{Funding}

This study was financially supported by the Science \& Technology Program of Tianjin First Central Hospital, Nankai University (Grant Nos. CM201803 and CF201807).

\section{Authors' contributions}

BY, XPL, TJ, and WDZ conceived of the study. HGZ, TX, and XHL performed data analysis for experiments. BY, HGZ, $T X$, and $L L$ drafted the final version of the manuscript and figure legends. BY, XPL, XHL, and LZ revised the figures, added critical content to the discussion, and were responsible for revising all portions of the submitted portion of the manuscript. TX and XHL performed the experiments using lung adenocarcinoma and control tissue. All contributors meet the criteria for authorship. All of the authors read and approved the final manuscript.

\section{Ethical approval and consent to participate}

This study was conducted in accordance with the Declaration of Helsinki and was confirmed by the Ethics Committee of Tianjin First Central Hospital of Nankai University (Tianjin, China; approval no. 2018N054KY). All patients signed the written informed consent forms.

\section{Consent for publication}

Not applicable

\section{Conflicts of Interest}

The authors declare that there are no conflicts of interest.

\section{Availability of data and materials}

The data of the current research are available from the corresponding author on a reasonable request.

\section{References}

1. Yoon HI, Kwon OR, Kang KN, Shin YS, Shin HS, Yeon EH, Kwon KY, Hwang I, Jeon YK, Kim Y et al: Diagnostic Value of Combining Tumor and Inflammatory Markers in Lung Cancer. Journal of cancer prevention 2016, 21(3):187-193.

2. Chalela R, Bellosillo B, Curull V, Longaron R, Pascual-Guardia S, Badenes-Bonet D, Arriola E, Sanchez-Font A, Pijuan L, Gea J: EGFR and KRAS Mutations in the Non-Tumoral Lung. Prognosis in Patients with Adenocarcinoma. Journal of clinical medicine 2019, 8(4).

3. Dhont L, Pintilie M, Kaufman E, Navab R, Tam S, Burny A, Shepherd F, Belayew A, Tsao MS, Mascaux C: Helicase-like transcription factor expression is associated with a poor prognosis in Non-Small-Cell Lung Cancer (NSCLC). BMC cancer 2018, 18(1):429. 
4. Zhang W, Li J, Li R, Zhang Y, Han M, Ma W: Efficacy and safety of iodine-125 radioactive seeds brachytherapy for advanced non-small cell lung cancer-A meta-analysis. Brachytherapy 2018, 17(2):439-448.

5. Chiu CH, Chou TY, Chiang CL, Tsai CM: Should EGFR mutations be tested in advanced lung squamous cell carcinomas to guide frontline treatment? Cancer chemotherapy and pharmacology 2014, 74(4):661-665.

6. Park JY, Jang SH, Kim HI, Kim JH, Park S, Hwang YI, Jung KS, Seo J, Lee CY, Ko Y et al: Thyroid transcription factor-1 as a prognostic indicator for stage IV lung adenocarcinoma with and without EGFR-sensitizing mutations. BMC cancer 2019, 19(1):574.

7. Liu WS, Zhao LJ, Pang QS, Yuan ZY, Li B, Wang P: Prognostic value of epidermal growth factor receptor mutations in resected lung adenocarcinomas. Medical oncology 2014, 31(1):771.

8. Hidayat M, Mitsuishi Y, Takahashi F, Tajima K, Yae T, Miyahara K, Hayakawa D, Winardi W, Ihara H, Koinuma Y et al: Role of FBXW7 in the quiescence of gefitinib-resistant lung cancer stem cells in EGFR-mutant non-small cell lung cancer. Bosnian journal of basic medical sciences 2019, 19(4):355-367.

9. Huang X, Xia L, Lan F, Shao YW, Li W, Xia Y: Treatment of Nivolumab Results in Hyperprogressive Disease in a Patient Harboring EGFR Exon 20 Insertion and MYC Amplification. Journal of thoracic oncology : official publication of the International Association for the Study of Lung Cancer 2019, 14(9):e189-e191.

10. Boulkroun S, Fay M, Zennaro MC, Escoubet B, Jaisser F, Blot-Chabaud M, Farman N, Courtois-Coutry N: Characterization of rat NDRG2 (N-Myc downstream regulated gene 2), a novel early mineralocorticoid-specific induced gene. The Journal of biological chemistry 2002, 277(35):31506-31515.

11. Guo Y, Li X, Sun X, Wang J, Yang X, Zhou X, Liu X, Liu W, Yuan J, Yao L et al: Combined Aberrant Expression of NDRG2 and LDHA Predicts Hepatocellular Carcinoma Prognosis and Mediates the Anti-tumor Effect of Gemcitabine. International journal of biological sciences 2019, 15(9):1771-1786.

12. Li SJ, Wang WY, Li B, Chen B, Zhang B, Wang X, Chen CS, Zhao QC, Shi H, Yao L: Expression of NDRG2 in human lung cancer and its correlation with prognosis. Medical oncology 2013, 30(1):421.

13. Wang XF, Wu YH, Wang MS, Wang YS: CEA, AFP, CA125, CA153 and CA199 in malignant pleural effusions predict the cause. Asian Pacific journal of cancer prevention : APJCP 2014, 15(1):363-368.

14. Jiang ZF, Wang M, Xu JL: Thymidine kinase 1 combined with CEA, CYFRA21-1 and NSE improved its diagnostic value for lung cancer. Life sciences 2018, 194:1-6.

15. Vervoorts J, Luscher-Firzlaff J, Luscher B: The ins and outs of MYC regulation by posttranslational mechanisms. The Journal of biological chemistry 2006, 281(46):34725-34729.

16. Liu J, Levens D: Making myc. Current topics in microbiology and immunology 2006, 302:1-32.

17. Lee EB, Kim A, Kang K, Kim H, Lim JS: NDRG2-mediated Modulation of SOCS3 and STAT3 Activity Inhibits IL10 Production. Immune network 2010, 10(6):219-229.

18. Wang H, Wang W, Wang X, Cai K, Wu H, Ju Q, Huang Z, Gao X: Reduced N-Myc downstream-regulated gene 2 expression is associated with CD24 upregulation and poor prognosis in patients with lung adenocarcinoma. Medical oncology 2012, 29(5):3162-3168.

19. Shimada K, Serada S, Fujimoto M, Nomura S, Nakatsuka R, Harada E, Iwahori K, Tachibana I, Takahashi T, Kumanogoh A et al: Molecular mechanism underlying the antiproliferative effect of suppressor of cytokine signaling-1 in non-small-cell lung cancer cells. Cancer science 2013, 104(11):1483-1491.

20. Wang B, Wang Z, Han L, Gong S, Wang Y, He Z, Feng Y, Yang Z: Prognostic significance of cyclin D3 expression in malignancy patients: a meta-analysis. Cancer cell international 2019, 19:158. 
21. Gao Y, Song P, Li H, Jia H, Zhang B: Elevated serum CEA levels are associated with the explosive progression of lung adenocarcinoma harboring EGFR mutations. BMC cancer 2017, 17(1):484.

22. Matsuoka K, Sumitomo S, Nakashima N, Nakajima D, Misaki N: Prognostic value of carcinoembryonic antigen and CYFRA21-1 in patients with pathological stage I non-small cell lung cancer. European journal of cardiothoracic surgery : official journal of the European Association for Cardio-thoracic Surgery 2007, 32(3):435-439.

23. Ji W, Qiu C, Wang M, Mao N, Wu S, Dai Y: Hsa_circ_0001649: A circular RNA and potential novel biomarker for colorectal cancer. Biochemical and biophysical research communications 2018, 497(1):122-126.

24. Fan G, Zhang K, Ding J, Li J: Prognostic value of EGFR and KRAS in circulating tumor DNA in patients with advanced non-small cell lung cancer: a systematic review and meta-analysis. Oncotarget 2017, 8(20):3392233932.

25. Mao C, Yuan JQ, Yang ZY, Fu XH, Wu XY, Tang JL: Blood as a Substitute for Tumor Tissue in Detecting EGFR Mutations for Guiding EGFR TKIs Treatment of Nonsmall Cell Lung Cancer: A Systematic Review and MetaAnalysis. Medicine 2015, 94(21):e775.

26. Kwak Y, Yun S, Nam SK, Seo AN, Lee KS, Shin E, Oh HK, Kim DW, Kang SB, Kim WH et al: Comparative analysis of the EGFR, HER2, c-MYC, and MET variations in colorectal cancer determined by three different measures: gene copy number gain, amplification status and the 2013 ASCO/CAP guideline criterion for HER2 testing of breast cancer. Journal of translational medicine 2017, 15(1):167.

27. Li WY, Zhao TT, Xu HM, Wang ZN, Xu YY, Han Y, Song YX, Wu JH, Xu H, Yin SC et al: The role of EGFR mutation as a prognostic factor in survival after diagnosis of brain metastasis in non-small cell lung cancer: a systematic review and meta-analysis. BMC cancer 2019, 19(1):145.

\section{Tables}

Table 1. Patients' demographic characteristics. 


\begin{tabular}{|c|c|c|}
\hline Clinical characteristics & Total n & $\%$ \\
\hline \multicolumn{3}{|l|}{ Age, years } \\
\hline$\leq 65$ & 42 & 47.2 \\
\hline$>65$ & 47 & 52.8 \\
\hline \multicolumn{3}{|l|}{ Sex } \\
\hline Male & 52 & 58.4 \\
\hline Female & 37 & 41.6 \\
\hline \multicolumn{3}{|l|}{ Smoking status } \\
\hline Non-smoker & 41 & 46.1 \\
\hline Smoker & 48 & 53.9 \\
\hline \multicolumn{3}{|l|}{ Blood type } \\
\hline A & 29 & 32.6 \\
\hline B & 27 & 30.3 \\
\hline $\mathrm{O}$ & 24 & 27.0 \\
\hline $\mathrm{AB}$ & 9 & 10.1 \\
\hline \multicolumn{3}{|l|}{ Lobe location } \\
\hline Right & 52 & 58.4 \\
\hline Upper lobe of right lung & 30 & 57.7 \\
\hline Middle lobe of right lung & 5 & 9.6 \\
\hline Inferior lobe of right lung & 12 & 23.1 \\
\hline Center-type of right lung & 5 & 9.6 \\
\hline Left & 37 & 41.6 \\
\hline Upper lobe of left lung & 21 & 56.8 \\
\hline Inferior lobe of left lung & 13 & 35.1 \\
\hline Center-type of left lung & 3 & 8.1 \\
\hline \multicolumn{3}{|l|}{$\mathrm{T}$} \\
\hline $1 \mathrm{a}$ & 9 & 10.1 \\
\hline $1 b$ & 11 & 12.4 \\
\hline $1 \mathrm{c}$ & 18 & 20.2 \\
\hline $2 \mathrm{a}$ & 21 & 23.6 \\
\hline $2 \mathrm{~b}$ & 13 & 14.6 \\
\hline 3 & 7 & 7.9 \\
\hline 4 & 10 & 11.2 \\
\hline \multicolumn{3}{|l|}{$\mathrm{N}$} \\
\hline 0 & 29 & 32.6 \\
\hline 1 & 20 & 22.5 \\
\hline 2 & 27 & 30.3 \\
\hline 3 & 13 & 14.6 \\
\hline \multicolumn{3}{|l|}{ M } \\
\hline 0 & 49 & 55.1 \\
\hline $1 \mathrm{a}$ & 20 & 22.5 \\
\hline $1 b$ & 6 & 6.7 \\
\hline $1 \mathrm{c}$ & 14 & 15.7 \\
\hline \multicolumn{3}{|l|}{ Stage } \\
\hline I & 24 & 27.0 \\
\hline II & 15 & 16.9 \\
\hline III & 10 & 11.2 \\
\hline IV & 40 & 44.9 \\
\hline \multicolumn{3}{|l|}{ Vascular invasion } \\
\hline No & 38 & 42.7 \\
\hline Yes & 51 & 57.3 \\
\hline \multicolumn{3}{|l|}{ EGFR } \\
\hline positive & 31 & 34.8 \\
\hline
\end{tabular}

Page $11 / 18$ 
T, tumor; $\mathrm{N}$, node; $\mathrm{M}$, metastasis; EGFR, epidermal growth factor receptor

Table 2. Patients' clinicopathological characteristics according to NDRG2 level.

\begin{tabular}{|c|c|c|c|c|c|c|}
\hline \multirow[b]{2}{*}{ Parameters } & \multicolumn{3}{|c|}{ Total patients $(n=89)$} & \multicolumn{3}{|c|}{ Operated patients $(\mathrm{n}=34)$} \\
\hline & $\begin{array}{l}\text { NDRG2 low } \\
\text { group }\end{array}$ & $\begin{array}{l}\text { NDRG2 high } \\
\text { group }\end{array}$ & P-value & $\begin{array}{l}\text { NDRG2 low } \\
\text { group }\end{array}$ & $\begin{array}{l}\text { NDRG2 high } \\
\text { group }\end{array}$ & value ${ }^{\mathrm{P}-}$ \\
\hline Gender & & & 0.988 & & & 0.710 \\
\hline Male & 31 & 21 & & 6 & 15 & \\
\hline Female & 22 & 15 & & 5 & 8 & \\
\hline Age (years) & & & 0.996 & & & 0.705 \\
\hline$<65$ & 25 & 17 & & 3 & 9 & \\
\hline$\geq 65$ & 28 & 19 & & 8 & 14 & \\
\hline Smoking status & & & 0.771 & & & 0.717 \\
\hline Non-smoker & 19 & 14 & & 5 & 13 & \\
\hline Smoker & 34 & 22 & & 6 & 10 & \\
\hline Blood type & & & 0.265 & & & 0.905 \\
\hline A & 15 & 14 & & 5 & 9 & \\
\hline B & 20 & 7 & & 1 & 4 & \\
\hline $\mathrm{AB}$ & 4 & 5 & & 2 & 5 & \\
\hline $\mathrm{O}$ & 14 & 10 & & 3 & 5 & \\
\hline Stage & & & $<0.001$ & & & 0.072 \\
\hline I & 6 & 18 & & 5 & 16 & \\
\hline II & 6 & 9 & & 3 & 7 & \\
\hline III & 8 & 2 & & 2 & 0 & \\
\hline IV & 33 & 7 & & 1 & 0 & \\
\hline & & & $<0.001$ & & & 0.028 \\
\hline $\mathrm{I}+\mathrm{II}$ & 12 & 27 & & 8 & 23 & \\
\hline III+IV & 41 & 9 & & 3 & 0 & \\
\hline CEA & & & $<0.001$ & & & $<0.001$ \\
\hline$<2.0$ & 4 & 24 & & 0 & 14 & \\
\hline$\geq 2.0$ & 49 & 12 & & 11 & 9 & \\
\hline $\begin{array}{l}\text { Vascular } \\
\text { invasion }\end{array}$ & & & $<0.001$ & & & 0.008 \\
\hline No & 19 & 32 & & 6 & 22 & \\
\hline Yes & 34 & 4 & & 5 & 1 & \\
\hline EGFR & & & 0.001 & & & 0.030 \\
\hline Negative(-) & 42 & 16 & & 9 & 9 & \\
\hline Positive(+) & 11 & 20 & & 2 & 14 & \\
\hline
\end{tabular}


Table 3. Prognostic value of conjoined expression of NDRG2/EGFR by multivariate Cox regression analysis.

\begin{tabular}{|c|c|c|c|c|c|c|}
\hline & \multirow[t]{2}{*}{ B } & \multirow[t]{2}{*}{ SE } & \multirow[t]{2}{*}{ P-value } & \multirow[t]{2}{*}{ HR } & \multicolumn{2}{|c|}{$95.0 \%$ CI for $\operatorname{Exp}(B)$} \\
\hline & & & & & Lower & Upper \\
\hline Age & 0.080 & 0.261 & 0.760 & 0.923 & 0.554 & 1.539 \\
\hline Sex & -0.171 & 0.317 & 0.590 & 1.186 & 0.637 & 2.209 \\
\hline Smoking status & 0.066 & 0.309 & 0.831 & 0.936 & 0.511 & 1.716 \\
\hline Lobe location & 0.186 & 0.262 & 0.479 & 0.831 & 0.497 & 1.388 \\
\hline Vascular invasion & 1.500 & 0.342 & $<0.001$ & 4.480 & 2.291 & 8.760 \\
\hline NDRG2-low/EGFR(-) & & & $<0.001$ & & & \\
\hline NDRG2-low/EGFR(+) & 1.873 & 0.464 & $<0.001$ & 6.508 & 2.619 & 16.174 \\
\hline NDRG2-high/EGFR(-) & 0.576 & 0.556 & 0.300 & 1.779 & 0.598 & 5.292 \\
\hline NDRG2-high/EGFR(+) & 1.258 & 0.476 & 0.008 & 3.519 & 1.384 & 8.949 \\
\hline
\end{tabular}

Figures

\begin{tabular}{|l|l|l|}
\hline $\begin{array}{l}\text { LUAD patients } \\
\text { Jun,2013-Jun,2014 }\end{array}$ & $\begin{array}{l}\text { PET/CT } \\
\text { Cardiac } \\
\text { ultrasonography } \\
\text { Lung function }\end{array}$ & \multicolumn{1}{|l|}{$\begin{array}{l}\text { LUAD patients enrolled } \\
(\mathrm{n}=89)\end{array}$} \\
\cline { 1 - 2 } & $\begin{array}{l}\text { Exclude secondary lung } \\
\text { cancer \& systemic } \\
\text { disease }\end{array}$ \\
\hline
\end{tabular}

Figure 1

Patient flowchart. Abbreviations: LUAD lung adenocarcinoma; PET positron-emission tomography; CT computed tomography; 125 I iodine-125 




D

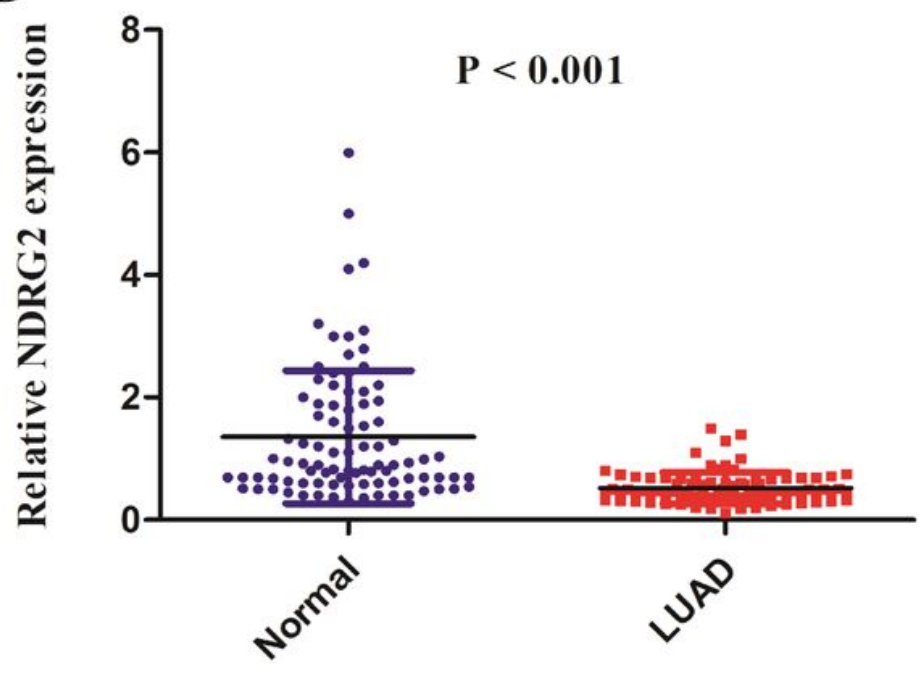

$\mathbf{E}$

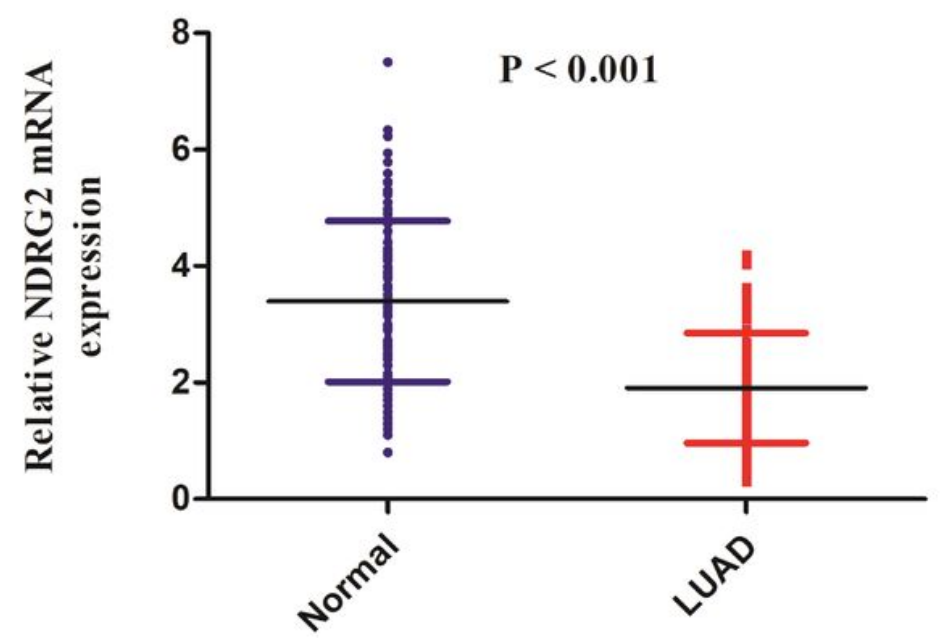

Figure 2

The expression level of NDRG2 in LUAD patients and normal tissues (Figure 2A-E). Immunohistochemistry showed the expression level of NDRG2 in normal lung tissues (Figure 2A), LUAD patients (Figure 2B), and negative control (Figure 2C) (200x magnification). The expression level of NDRG2 protein was determined by western blot assay (Figure 2D) and RT-PCR (Figure 2E). It was significantly downregulated in patients with LUAD compared with that in normal tissues at both protein and mRNA levels ( $\left.{ }^{*} P<0.001\right)$. Abbreviations: NDRG2, N-Myc downstreamregulated gene 2; LUAD, lung adenocarcinoma; RT-PCR, reverse transcription-polymerase chain reaction. 
A

\section{Normal}

I

II

III

IV


GAPDH $\square \longrightarrow C$

C



B

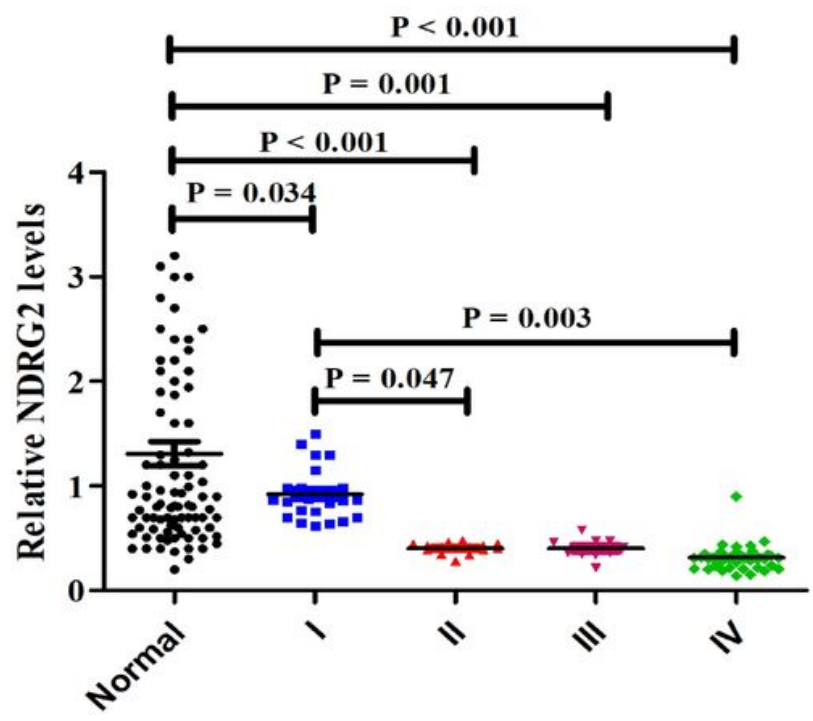

D
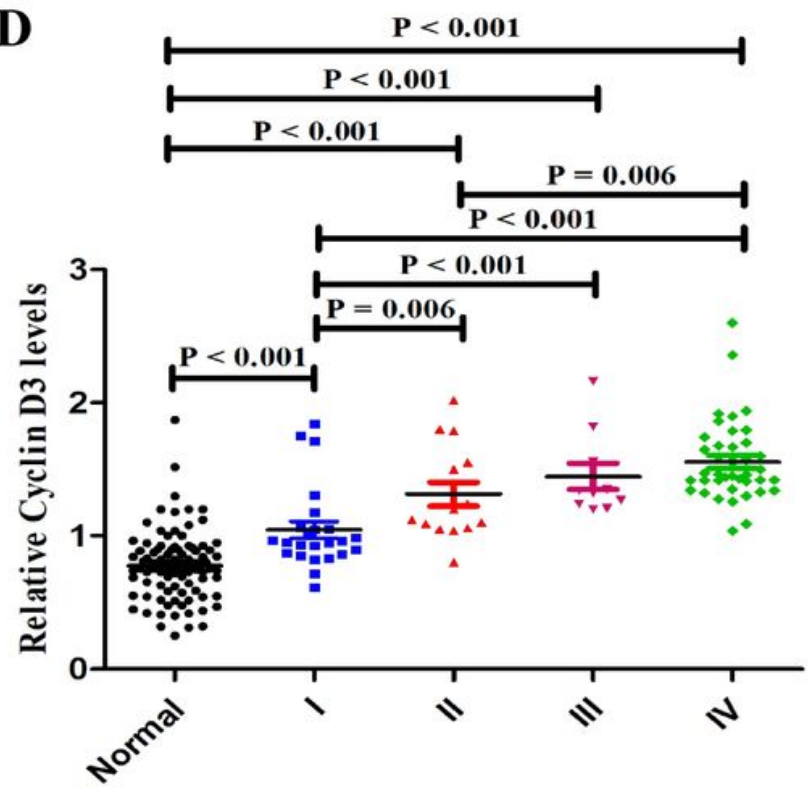

Figure 3

Associations between the expression levels of NDRG2 and clinicopathological features of patients with LUAD. The NDRG2, SOCS1, and CyclinD3 levels were evaluated by western blot (Figure 3A-D). The NDRG2 and SOCS1 levels decreased with the increasing LUAD stage, while the cyclinD3 levels increased with the increasing LUAD stage. Abbreviations: NDRG2, N-Myc downstream-regulated gene 2; LUAD, lung adenocarcinoma; SOCS1, suppressor of cytokine signaling 1; GAPDH: Glyceraldehyde-3-phosphate dehydrogenase 


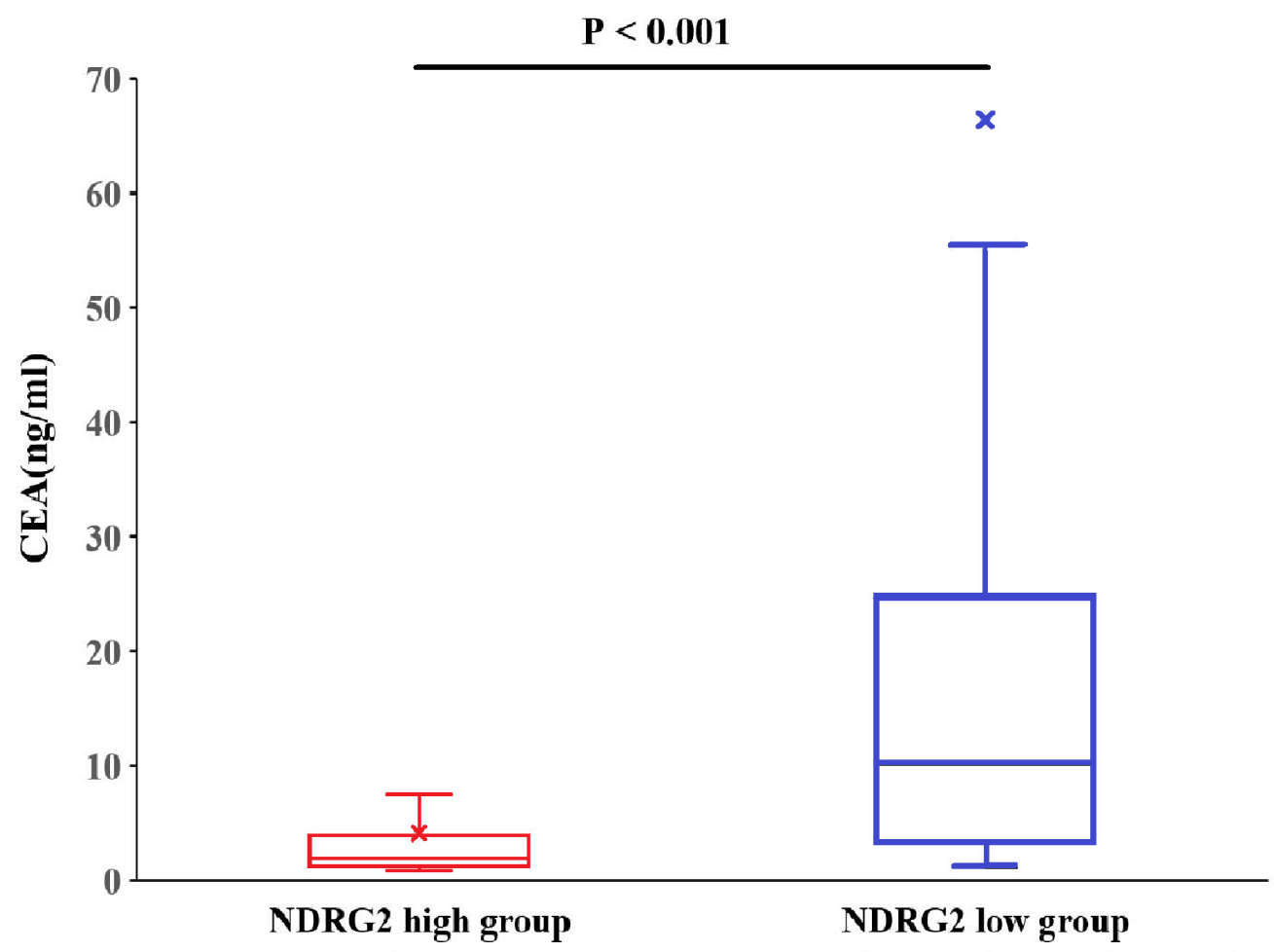

Figure 4

Associations between the expression levels of NDRG2 and CEA levels of patients with LUAD. The CEA levels were notably lower in patients with a high expression level of NDRG2 than that in patients with the low expression level of NDRG2 (P < 0.001). Abbreviations: CEA, carcinoembryonic antigen; NDRG2, N-Myc downstream-regulated gene 2; LUAD, lung adenocarcinoma. 
A



B



Time after iodine-125 implantation (months)

C

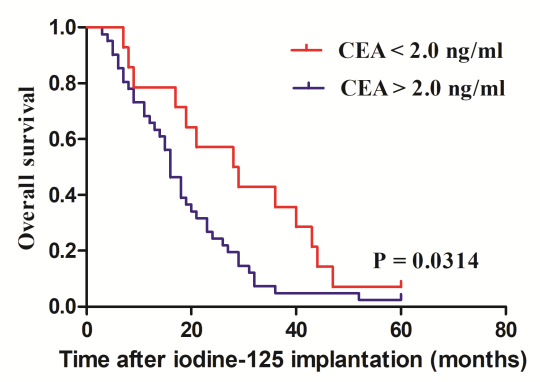

D

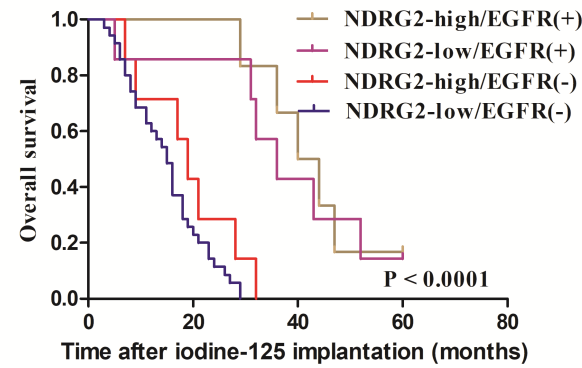

$\mathbf{E}$

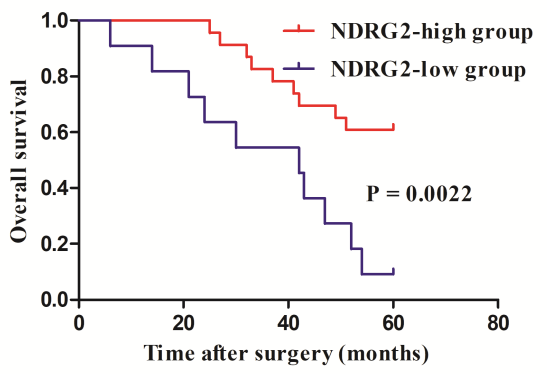

F

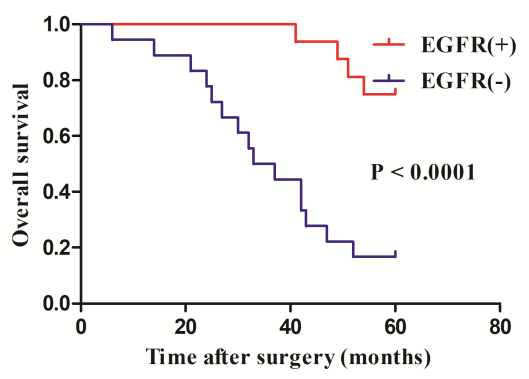

G

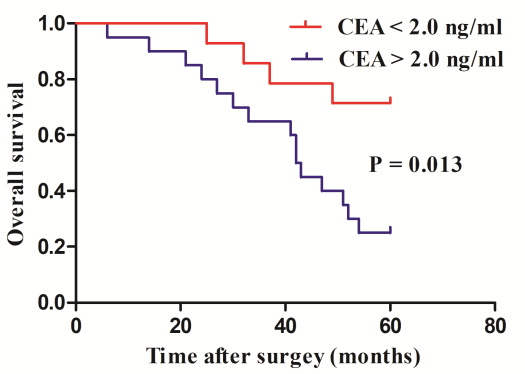

H

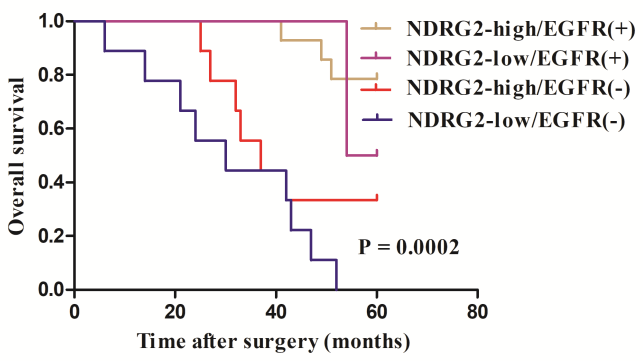

Figure 5

Overall survival of patients with LUAD (Figure 3A-H). Overall survival of patients with advanced LUAD treated with iodine-125 radioactive seeds brachytherapy (Figure 3A-D). The patients with low expression level of NDRG2 ( $\mathrm{P}=$ 0.0261, Figure 3A), negative EGFR expression ( $P<0.0001$, Figure 3B), and CEA $>2.0 \mathrm{ng} / \mathrm{ml}(\mathrm{P}=0.0314$, Figure 3C) exhibited significantly lower overall survival rates. According to the conjoined expressions of NDRG2/EGFR, the subjects were categorized into four groups: NDRG2-low/EGFR-negative(-), NDRG2-low/EGFR-positive(+), NDRG2high/EGFR-negative(-), and NDRG2-high/EGFR-positive(+). Patients with co-expression of NDRG2-low/EGFRnegative(-) had the worst outcome of overall survival among the four groups $(P<0.0001$, Figure 3D). Overall survival of operated patients (Figure 3E-H). The patients with low expression level of NDRG2 $(P=0.0022$, Figure 
3E), negative EGFR expression ( $P<0.0001$, Figure 3F), and CEA $>2.0 \mathrm{ng} / \mathrm{ml}(P=0.013$, Figure 3G) exhibited remarkably lower overall survival rates. Patients with co-expression of NDRG2-low/EGFR-negative had the worst outcome for overall survival among the four groups $(P=0.0002$, Figure $3 H)$. Abbreviations: LUAD, lung adenocarcinoma; NDRG2, N-Myc downstream-regulated gene2; CEA, carcinoembryonic antigen; EGFR, epidermal growth factor receptor.

\section{Supplementary Files}

This is a list of supplementary files associated with this preprint. Click to download.

- originalSOCS1.jpg

- originalCyclinD3GAPDH.jpg

- originalNDRG2.jpg 